\title{
Association of Circulating Circular RNAs (mmu-015947, hg38-0008980, and DLGAP4) in Diagnosis, Diseases Severity, and Prognosis of Ischemic Stroke
}

\author{
Mastaneh Zeraatiannejad \\ Islamic Azad University \\ Mahnaz Bayat \\ Shiraz University of Medical Sciences \\ Reza Tabrizi \\ Fasa University of Medical Science \\ Afshin Borhani-Haghighi \\ Shiraz University of Medical Sciences \\ Mohammad Javad Mokhtari ( $\nabla$ mj.mokhtari@gmail.com ) \\ Islamic Azad University https://orcid.org/0000-0001-5066-9632
}

\section{Research Article}

Keywords: Circular RNAs, mmu-015947, hg38-0008980, CircDLGAP4, Ischemic stroke

Posted Date: December 16th, 2021

DOI: https://doi.org/10.21203/rs.3.rs-1152366/v1

License: (c) (i) This work is licensed under a Creative Commons Attribution 4.0 International License. Read Full License 


\section{Abstract}

Backgrounds Quickly diagnosing ischemic stroke (IS) is a critical issue in clinical studies, as it allows more effective therapy and stops the progression of IS. The blood level of CircRNAs after stroke may act as a rapid diagnostic marker.

Methods and Results In this study, the blood level of circRNAs was evaluated using a real-time PCR. We used logistic and linear regression analysis to evaluate the potential of circRNAs levels with the risk of IS.

Circ-mmu-015947 was up-regulated in patients. Its expression also showed a good value in predicting IS risk, and a significant diagnostic value. CircDLGAP4 was decreased in patients compared with controls and logistic regression showed its expression negatively associated with IS risk. The expression level of hg38 was reduced significantly in patients with small vessel disease (SVD) and the linear regression analysis showed a negative relationship between hg38 expression with SVD subtype. Circhg38 expression relative to controls showed a significant association with IS risk.

Conclusion Taken together, circulating circ-mmu-015947 may serve as a novel biomarker for IS, and we found a significant decrease in the level of hg38-0008980 after IS it may act as a novel circRNA in IS pathophysiology with a positive correlation with stroke severity.

\section{Introduction}

Stroke is the most common cause of morbidity, mortality, and long-term disability in developed and developing countries $[1,2]$. There are several mimics and camouflages for ischemic stroke (IS) [3]. Thus, finding the rapid diagnostic test to confirm the clinical diagnosis and more effective therapy of ischemic stroke is necessary [4].

One of the endogenous single-stranded RNAs with a closed covalent-circle structure is circular RNAs (circRNAs) [5]. They are produced during post-transcriptional modification by back-splicing of mRNAs or IncRNAs and because of their covalent structure as well as resistance to exonuclease are much more stable than linear RNA thus can serve as a diagnostic marker in the peripheral blood of patients [6]. CircRNAs can regulate the expression of specific target genes. Several circRNAs are involved in the regulation of ischemic-reperfusion injury (IRI) $[7,8]$ and other cerebral diseases $[9,10]$. CircRNAs may also act as a diagnostic biomarker for discriminating neurological disorders [11] while they mainly have a sponge function for the regulation of microRNA (miRNA) [12]. Animal studies have revealed a close association of cerebral circRNA expression with IS $[7,13,14]$.

Bioinformatics analysis by Lin et al. revealed that the up-regulated expression of mmu-015947 in HT22 cells with oxygenglucose deprivation/reoxygenation (OGD/R) could interact with miRNAs and may participate in metabolism, apoptosis, and immune-related pathways. According to the important roles of these pathways in the pathogenesis of IR, Lin et al. suggested that the up-regulation of mmu-015947 might be involved in the process of cerebral IRI [7]. Therefore, we presumed that the circ-mmu-015947 may function as a significant diagnostic biomarker in IS patients. The circDLGAP4 is a sponge for miR-143 and in animal models has been shown that the overexpression of circDLGAP4 attenuated the neurological deficits, the infarct areas, and blood-brain damage [8]. Bai et al. has shown that circDLGAP4 levels were significantly decreased in the acute IS patients. They demonstrated that circDLGAP4 could inhibit miR-143 activity [8]. Silencing of miR-143 exerted a protective effect against cerebrovascular damage [15].

The circDLGAP4-miR-143 network by exerting certain important regulatory effects on the permeability of the blood-brain barrier (BBB) attenuates IS outcome [16, 17].

Another circRNA that has shown the important regulatory effect on BBB integrity is hg38-circ-0008980. It is a novel circRNAs could sponge hsa-miR-660-5p and exert regulatory effects of meningitic E. coli penetration on BBB [18]. 
According to the above-mentioned circ hg38-0008980 is likely to have a regulatory effect on BBB permeability in IS. There is no evidence about hg38-0008980 expression after IS. Thus we hypothesized that the expression of circ hg38-0008980 may alter after IS and serve as a significant diagnostic marker for IS. Therefore, we evaluated for the first time the expression levels of two novel circRNAs (mmu-015947, hg38-0008980) and also circDLGAP4 level in peripheral blood of 75 IS patients and 75 controls by real-time PCR, to determine their expression levels within the first 24 hours after IS to correlate these levels with the stroke severity, clinical parameters as well as their possible application for diagnosis and prognosis of IS.

\section{Methods And Materials Study subjects}

This is a case-control study conducted at Namazi Hospital in Shiraz. All patients underwent brain diffusion-weighted magnetic resonance imaging (MRI) or non-contrast computed tomography (CT), to differentiate IS from intracerebral hemorrhage. According to the Guidelines, stroke is an acute neurologic disorder lasting more than 24 hours (or $<24$ hours with neuroimaging evidence) [19]. In this study, inclusion criteria were as follows: patients must be admitted within $24 \mathrm{~h}$ from the onset of stroke without a history of the previous stroke, older than 18 years, and we could follow them regularly. We excluded patients who suffered from different types of cancer, treated with an immunosuppressant, severe inflammation, and patients who were pregnant or breastfeeding, patients with the transient ischemic attack (TIA) were excluded. 75 controls from Shiraz's population randomly were included in our study while were matched for sex and age with cases. The ages of the cases and controls ranged from 30-95 years. Controls with a history of stroke, TIA, or severe brain disorders were excluded. Figure 1 shows the criteria for screening, exclusion and enrolment of the study participants.

In this study, hypertension and diabetes were diagnosed according to these criteria: for hypertension: receiving antihypertensive therapy or blood pressure $\geq 140 / 90 \mathrm{mmHg}$ on two occasions at least $24 \mathrm{~h}$ apart [20]. Diabetes mellitus: receiving treatment with hypoglycemic drugs or two fasting glucose $>126 \mathrm{mg} / \mathrm{dl}(7.0 \mathrm{mmol} / \mathrm{L})$ and 2-h post-load glucose $>200 \mathrm{mg} / \mathrm{dl}(11.1 \mathrm{mmol} / \mathrm{L})$ or [21]. To indicate the severity of stroke we used the National Institutes of Health Stroke Scale (NIHSS) score on admission, higher scores represent greater severity [22]. Six months after admission the functional outcomes were obtained according to the modified Rankin scale (mRS) blinded to the level of CircRNA [23]. Different types of IS were diagnosed according to the TOAST classification [24]. Ethics approval for this study was obtained by the local ethics committee of the Arsanjan Branch, Islamic Azad University, Iran (IR.IAU.A.REC.1399.029). Peripheral venous blood samples were collected after taking written informed consent from all subjects (or their proxy respondents).

\section{Measurement of the circRNAs levels}

TRIzol Reagent (GeneAll, Seoul, South Korea) was used for the isolation of total RNA from blood samples according to the thiocyanate-phenol-chloroform method. Using cDNA synthesis Kit (Yektatajhiz, Iran) for cDNA synthesis from RNA samples with the A260/A230 and A260/A280 ratios greater than 1.7. We used quantitative real-time PCR for mmu-015947, circDLGAP4, and hg38-0008980 levels measurement using RealQ Plus 2x Master Mix Green Low ROX ${ }^{\mathrm{TM}}$ (Ampliqon, Denmark). TBP was used as an internal reference for circRNAs detection. The Quantstudio 3 Real-Time PCR System (Applied Biosystems, Foster City, USA) was used while the thermal-cycling settings were 1 repeat (10 min at $95^{\circ} \mathrm{C}$ ) accompanied by 40 cycles $\left(15 \mathrm{~s}\right.$ at $95^{\circ} \mathrm{C}, 30 \mathrm{~s}$ at $62^{\circ} \mathrm{C}, 45 \mathrm{~s}$ at $\left.72^{\circ} \mathrm{C}\right)$ and 1 cycle $\left(5 \mathrm{~min} 72^{\circ} \mathrm{C}\right)$. The melting phase was ( $15 \mathrm{~s}$ at $95^{\circ} \mathrm{C}, 30 \mathrm{~s}$ at $72^{\circ} \mathrm{C}, 15 \mathrm{~s}$ at $95^{\circ} \mathrm{C}$ ). Variation in expression levels was expressed using the cycle threshold (Ct) values. $\Delta \mathrm{Ct}$ represents the difference of $\mathrm{Ct}$ between TBP and the target gene. The relative circRNAs expression levels for every individual were defined using $2^{-\Delta C t}$. The results were calculated with the $2^{-\Delta C t}$ method [25].

\section{The primer sequences were used as follows:}


circ-mmu-015947:Forward:CGTGCATGAGTGAGGAGACCA,Reverse ATGGGATGCTTCGTGGGCCTT.hg38-circ-

0008980:Forward : CTCCACCAGATGTCAGTT Reverse: TACAGGCAGAGGGTATTTG. TATA box-binding protein (TBP, reference gene), 5'-CCCGAAACGCCGAATATAATC-3'(forward) and 5'-TCTGGACTGTTCTTCACTCTTG-3' (reverse).

\section{Statistical analysis}

The circRNAs expression levels were shown as mean \pm SE. The following tests were used for different analyses in our study, The student's t-test for comparing the mean expression of the circ-RNAs between case and control groups. One-Way ANOVA for comparing the circRNA expression levels between different types of stroke. A comparison between categorical data was carried out using a chi-square test. The expression level of circRNAs was compared between cases and controls using an independent two-sample t-test. We used logistic regression analyses to evaluate the association of circRNA expression with the risk of IS. relationship between circRNA levels with clinical parameters was analyzed using linear regression. By ROC curve analysis the diagnostic and prognostic values were estimated and the findings were presented as the area under the curve (AUC). Correlations were analyzed using the Spearman correlation. The analyses were done using the SPSS software (version 19.0) and GraphPad Prism 5.0. The $p$-value of $<0.05$ was regarded as statistically significant.

\section{Results}

\section{Demographic and clinical characteristics of all participants (IS patients and controls)}

The control group was similar in age, sex, and BMI to the case group (Table 1). Risk factors such as diabetes, hypertension, and smoking were demonstrated to be more prevalent in cases compared to controls. In laboratory findings, there were no significant differences in the levels of triglyceride and total cholesterol between the IS cases and the controls; however, low-density lipoprotein was significantly higher and high-density lipoprotein levels were significantly lower in IS cases compared to controls (Table 1). 
Table 1

Demographic and clinical characteristics of the participants.

\begin{tabular}{|c|c|c|c|c|}
\hline \multicolumn{2}{|l|}{ Characteristics } & Cases $(n=75)$ & Controls $(n=75)$ & $p$ \\
\hline \multicolumn{2}{|l|}{ Male, n (\%) } & 48 (64\%) & $48(64 \%)$ & \multirow[t]{2}{*}{$0.999^{a}$} \\
\hline \multicolumn{2}{|l|}{ Female, n (\%) } & 27 (36\%) & 27 (36\%) & \\
\hline \multicolumn{2}{|l|}{ Age (years) } & $64.79 \pm 1.78$ & $64.55 \pm 1.68$ & $0.922^{b}$ \\
\hline \multicolumn{2}{|l|}{$\mathrm{BMI}\left(\mathrm{kg} / \mathrm{m}^{2}\right)$} & $25.64 \pm 0.53$ & $25.99 \pm 0.45$ & $0.619^{b}$ \\
\hline \multicolumn{2}{|l|}{ NIHSS at admission } & $30(40 \%)$ & & 0.00 \\
\hline \multicolumn{2}{|l|}{$\leq 6$} & $45(60 \%)$ & & \\
\hline \multicolumn{5}{|l|}{$\geq 7$} \\
\hline \multicolumn{2}{|l|}{$\mathrm{mRS}$ at 3 months at admission } & $23(30.66 \%)$ & & 0.00 \\
\hline \multicolumn{2}{|l|}{$0-2$} & $52(96.33 \%)$ & & \\
\hline \multicolumn{5}{|l|}{$3-6$} \\
\hline \multicolumn{2}{|l|}{ mRS at 6 months } & $36(48 \%)$ & & 0.00 \\
\hline \multicolumn{2}{|l|}{$0-2$} & $39(52 \%)$ & & \\
\hline \multicolumn{5}{|l|}{$3-6$} \\
\hline \multicolumn{5}{|l|}{ Vascular risk factors } \\
\hline \multirow[t]{2}{*}{ Hypertension, n (\%) } & Yes & 35 (46.66\%) & 30 (13.33\%) & 0.641 \\
\hline & No & 40 (53.33\%) & $45(60 \%)$ & \\
\hline \multirow[t]{2}{*}{ Diabetes, n (\%) } & Yes & $18(24 \%)$ & $20(26.66 \%)$ & 0.452 \\
\hline & No & 57 (76\%) & $55(73.33 \%)$ & \\
\hline \multirow[t]{2}{*}{ Smoking, n (\%) } & Yes & $14(18.66 \%)$ & $3(4 \%)$ & $0.005^{a}$ \\
\hline & No & 61(81.33\%) & 72 (96\%) & \\
\hline \multirow[t]{2}{*}{ Drinking, n (\%) } & Yes & $3(4 \%)$ & $0(0 \%)$ & $0.080^{a}$ \\
\hline & No & $72(96 \%)$ & 75 (100\%) & \\
\hline \multirow[t]{2}{*}{ Hyperlipidemia } & Yes & $11(14.16 \%)$ & $8(10.66 \%)$ & $0.461^{a}$ \\
\hline & No & 64 (85.33\%) & 67(83.33\%) & \\
\hline \multicolumn{5}{|l|}{ Laboratory findings } \\
\hline \multicolumn{2}{|l|}{ Triglyceride, mmol/L } & $117.83 \pm 5.30$ & $122.73 \pm 7.58$ & $0.597^{b}$ \\
\hline \multicolumn{2}{|l|}{ Total Cholesterol, mmol/L } & $144.35 \pm 5.29$ & $114.89 \pm 4.37$ & $0.999^{b}$ \\
\hline \multicolumn{2}{|l|}{ Low Density Lipoprotein, mmol/L } & $91.25 \pm 3.209$ & $81.24 \pm 3.243$ & $0.030^{\mathrm{b}}$ \\
\hline \multicolumn{2}{|l|}{ High Density Lipoprotein, mmol/L } & $36.07 \pm 0.847$ & $41.41 \pm 1.265$ & $0.001^{b}$ \\
\hline
\end{tabular}




\begin{tabular}{|c|c|c|c|}
\hline Characteristics & Cases $(n=75)$ & Controls $(n=75)$ & $p$ \\
\hline Large artery atherosclerosis $\mathrm{n}(\%)$ & $30(40 \%)$ & & \\
\hline Small-vessel occlusion n (\%) & $26(34.66 \%)$ & & \\
\hline Cardio embolism n (\%) & $7(9.33 \%)$ & & \\
\hline Undetermined n (\%) & $12(16 \%)$ & & \\
\hline \multicolumn{4}{|c|}{ Data were shown as mean \pm SD or as $n(\%) .{ }^{a}$ Chi-square Test; ${ }^{b}$ Independent two-sample T-test. } \\
\hline \multicolumn{4}{|c|}{ BMI, body mass index; NIHSS, National Institutes of Health Stroke Scale; mRS, modified Rankin Scale. } \\
\hline
\end{tabular}

\section{Expression of mmu-015947 in IS patients was higher than controls}

In IS patients, mmu expression was shown higher than the controls at 0-24 hours after stroke (2.04 \pm 0.12 vs $0.97 \pm 0.09)$ $(p<0.0001$, Fig. 2A). Furthermore, we studied and compared the mmu-015947 expression level in 4 subtypes of IS, results showed the level of mmu-015947 in SVD $(p<0.0001)$, LAA $(p<0.0001)$, and UD IS $(p<0.01)$ were significantly higher than those in the controls. But no marked differences were found between the levels of mmu-015947 in the 4 subgroups $F$ ( 4 , $145)=11.48, p<0.0001$ (Fig. 2B). Moreover, logistic regression analysis was performed to test the association of CircRNA expression with the risk of IS. mmu level was significantly associated with risk of stroke (OR = 1.008, 95\% Cl: 1.78-4.19, $p<$ 0.0001) after adjusting for age, gender, and other risk factors. Hypertension was also significantly showed a positive association with the risk of stroke (OR $=1.54,95 \% \mathrm{Cl}: 1.74-12.5, p<0.002)$ (Data not shown).

Expression of hg38- 0008980 and circDLGAP4 in IS patients were lower than controls.

The blood levels of CircRNAs DLGAP4 and hg38 in IS patients were significantly lower than the controls at 0-24 h after stroke respectively $[(1.90 \pm 0.11$ vs $2.23 \pm 0.10)$ and $(1.71 \pm 0.13$ vs $2.37 \pm 0.24)](p<0.05$, Fig. $2 A)$. Furthermore, One-Way ANOVA analysis showed the level of DLGAP4 and hg38 in SVD cases were significantly lower than those in the controls $(p$ $<0.05$ ) (Fig. 2C and D) [DLGAP4; F $(4,145)=3.506, p=0.0092$ ] and [hg38; $F(4,145)=2.259, p=0.0656$ ]. However, a significant decrease in DLGAP4 expression was reported in patients with SVD relative to UD (Fig. 2C).

logistic regression analysis also revealed the significant negative association of hg38 and DLGAP4 expression with the risk of IS (adjusted OR $=-0.412 ; 95 \% \mathrm{Cl}: 0.49-0.88, p<0.01$ ) and (adjusted OR $=-0.404 ; 95 \% \mathrm{Cl}: 0.45-0.98, p<0.05$ ) respectively (Data not shown).

\section{Association between clinical variables with CircRNAs (mmu-015947, hg38-0008980, and DLGAP4) expression}

The association between clinical variables and the circRNAs expression was defined by subgroup analyses (Table 2). The levels of mmu expression were shown to be significantly higher in the positive hypertension subgroup $(p<0.01)$ and also revealed that the level of hg38 expression significantly was higher in patients with NIHSS scores $>7$. 
Table 2

The association between expression of circular RNAs with clinical parameters in IS patients by Subtype analysis.

\begin{tabular}{|c|c|c|c|c|c|c|c|c|c|c|}
\hline & & mmu- 0159 & & & DLGAP4 & & & hg38-0008s & & \\
\hline Characteristics & $\mathrm{n}$ & Mean $\pm S E$ & $\mathrm{t}$ & $\mathrm{p}$ & Mean $\pm S E$ & $t$ & $p$ & Mean士SE & $t$ & $p$ \\
\hline Gender & & & 0.371 & 0.711 & & & -0.27 & & 0.058 & 0.954 \\
\hline Male & 48 & $2.01 \pm 1.98$ & & & $1.88 \pm 0.141$ & & & $2.04 \pm 1.50$ & & \\
\hline Female & 27 & $2.13 \pm 1.92$ & & & $1.94 \pm 0.209$ & & & $2.05 \pm 2.08$ & & \\
\hline Age & & & 0.578 & 0.565 & & 0.001 & 0.999 & & 0.970 & 0.335 \\
\hline$<70$ & 45 & $3.33 \pm 1.33$ & & & $1.90 \pm 1.10$ & & & $1.61 \pm 1.20$ & & \\
\hline$\geq 70$ & 30 & $2.57 \pm 2.14$ & & & $1.90 \pm 0.88$ & & & $1.87 \pm 1.11$ & & \\
\hline BMI (kg/m2) & & & 0.957 & 0.342 & & 1.530 & 0.130 & & 0.167 & 0.868 \\
\hline$<24$ & 32 & $2.64 \pm 2.12$ & & & $2.11 \pm 1.05$ & & & $1.74 \pm 1.14$ & & \\
\hline$\geq 24$ & 43 & $2.26 \pm 1.30$ & & & $1.75 \pm 0.96$ & & & $1.69 \pm 1.20$ & & \\
\hline NIHSS & & & 0.049 & 0.961 & & 1.701 & 0.092 & & 2.118 & 0.038 \\
\hline$<6$ & 30 & $2.41 \pm 1.59$ & & & $1.66 \pm 1.13$ & & & $1.37 \pm 1.18$ & & \\
\hline$\geq 7$ & 45 & $2.43 \pm 1.77$ & & & $2.06 \pm 0.90$ & & & $1.94 \pm 1.11$ & & \\
\hline $\begin{array}{l}\mathrm{mRS} \text { at } 3 \\
\text { months }\end{array}$ & & & 0.713 & 0.478 & & 1.242 & 0.219 & & 0.897 & 0.382 \\
\hline $0-2$ & 10 & $2.21 \pm 1.86$ & & & $1.68 \pm 1.07$ & & & $1.89 \pm 1.28$ & & \\
\hline $3-6$ & 65 & $2.52 \pm 1.62$ & & & $2.00 \pm 0.98$ & & & $1.63 \pm 1.12$ & & \\
\hline $\begin{array}{l}\mathrm{mRS} \text { at } 6 \\
\text { months }\end{array}$ & & & 0.285 & 0.06 & & 0.812 & 0.422 & & 0.195 & 0.846 \\
\hline $0-2$ & 10 & $1.87 \pm 1.67$ & & & $1.80 \pm 1.00$ & & & $1.69 \pm 1.24$ & & \\
\hline $3-6$ & 65 & $2.94 \pm 1.56$ & & & $1.99 \pm 1.02$ & & & $1.74 \pm 1.11$ & & \\
\hline Hypertension & & & 3.431 & 0.001 & & -.033 & 0.392 & & 0.140 & 0.889 \\
\hline Negative & 40 & $1.70 \pm 1.44$ & & & $1.86 \pm 0.175$ & & & $2.03 \pm 1.82$ & & \\
\hline Positive & 35 & $2.86 \pm 2.66$ & & & $1.94 \pm 0.153$ & & & $2.07 \pm 1.48$ & & \\
\hline Diabetes & & & 1.011 & 0.314 & & 1.029 & 0.307 & & 0.396 & 0.692 \\
\hline Negative & 57 & $1.99 \pm 2.02$ & & & $1.97 \pm 0.130$ & & & $2.06 \pm 1.77$ & & \\
\hline Positive & 18 & $2.49 \pm 1.36$ & & & $1.69 \pm 0.261$ & & & $1.89 \pm 1.29$ & & \\
\hline Smoking & & & 0.508 & 0.612 & & -1.15 & 0.252 & & 0.539 & 0.591 \\
\hline Negative & 61 & $2.03 \pm 2.02$ & & & $1.84 \pm 0.129$ & & & $2.01 \pm 1.69$ & & \\
\hline Positive & 14 & $2.28 \pm 1.34$ & & & $2.18 \pm 0.264$ & & & $2.25 \pm 1.98$ & & \\
\hline
\end{tabular}

BMI, body mass index; NIHSS, National Institutes of Health Stroke Scale; mRS, modified Rankin Scale. Independent two-sample T-test. 


\begin{tabular}{|c|c|c|c|c|c|c|c|c|c|}
\hline & & mmu- 0159 & & & DLGAP4 & & & hg38-0008980 & \\
\hline Drinking & & & 0.279 & 0.781 & & -0.55 & 0.580 & 0.462 & 0.645 \\
\hline Negative & 72 & $2.04 \pm 1.97$ & & & $1.89 \pm 0.121$ & & & $2.03 \pm 1.73$ & \\
\hline Positive & 3 & $2.36 \pm 0.292$ & & & $2.22 \pm 0.249$ & & & $2.50 \pm 1.08$ & \\
\hline Hyperlipidemia & & & 0.463 & 0.644 & & 0.609 & 0.544 & 0.657 & 0.513 \\
\hline Negative & 64 & $2.08 \pm 2.03$ & & & $1.93 \pm 0.123$ & & & $2.01 \pm 1.72$ & \\
\hline Positive & 11 & $1.86 \pm 1.38$ & & & $1.73 \pm 0.360$ & & & $2.28 \pm 1.76$ & \\
\hline \multicolumn{10}{|c|}{ BMI, body mass index; NIHSS, National Institutes of Health Stroke Scale; mRS, modified Rankin Scale. } \\
\hline \multicolumn{10}{|c|}{ Independent two-sample T-test. } \\
\hline
\end{tabular}

Furthermore, we also used linear regression analysis to detect the association between the circRNA levels with clinical parameters and types of stroke. We found the significant negative relationships between hg38 expression and SVD subtype $(p=0.008$, Beta $=-0.036)$ and also between DLGAP4 level and BMI in IS patients $(p=0.02$, Beta $=-0.29)($ Table 3$)$.

Table 3

Linear regression analysis for the association between clinical parameters with CircRNAs mmu- 015947, DLGAP4, and hg38-0008980 in ischemic stroke patients.

\begin{tabular}{|c|c|c|c|c|c|c|c|c|c|c|c|c|}
\hline Variables & $\begin{array}{l}\text { Beta } \\
\text { mmu }\end{array}$ & $\begin{array}{l}95 \% \\
\mathrm{Cl}\end{array}$ & & $p$ & $\begin{array}{l}\text { Beta } \\
\text { DLGAP4 }\end{array}$ & $\begin{array}{l}95 \% \\
\mathrm{Cl}\end{array}$ & & $p$ & $\begin{array}{l}\text { Beta } \\
\text { hg38 }\end{array}$ & $\begin{array}{l}95 \% \\
\mathrm{Cl}\end{array}$ & & $p$ \\
\hline SEX & -.064 & -.732 & .435 & .613 & .076 & -.327 & .644 & .516 & -.155 & -.952 & .201 & .198 \\
\hline Age & -.200 & -.034 & .005 & .138 & -.125 & -.024 & .008 & .309 & .080 & -.013 & .025 & .527 \\
\hline BMI & -.111 & -.093 & .039 & .415 & -.292 & -.119 & -.009 & .022 & -.066 & -.081 & .048 & .609 \\
\hline NIHSS & .007 & -.045 & .047 & .957 & .038 & -.032 & .044 & .757 & .097 & -.028 & .063 & .440 \\
\hline MRS & .004 & -.204 & .211 & .973 & -.148 & -.281 & .064 & .214 & -.122 & -.308 & .102 & .319 \\
\hline HTN & .075 & -.393 & .730 & .551 & .104 & -.256 & .677 & .371 & .090 & -.345 & .765 & .453 \\
\hline DM & .133 & -.361 & 1.053 & .332 & -.033 & -.666 & .509 & .790 & .117 & -.381 & 1.01 & .367 \\
\hline SVD & -.008 & -.684 & .647 & .956 & -.150 & -.870 & .236 & .257 & -.367 & -1.55 & -.240 & .008 \\
\hline CE & -.108 & -1.42 & .593 & .414 & -.109 & -1.21 & .462 & .373 & -.095 & -1.37 & .618 & .451 \\
\hline UD & -.049 & -.953 & .655 & .712 & .215 & -.079 & 1.258 & .083 & -.197 & -1.42 & .169 & .121 \\
\hline \multicolumn{13}{|c|}{$\begin{array}{l}\text { BMI, body mass index; NIHSS, National Institutes of Health Stroke Scale; mRS, modified Rankin Scale DM, diabetes } \\
\text { mellitus; }\end{array}$} \\
\hline $\begin{array}{l}\text { IHD, Ische } \\
\text { cardioemb }\end{array}$ & $\begin{array}{l}\text { hear } \\
\text { sm;Ut }\end{array}$ & $\begin{array}{l}\text { sease } \\
\text { idete }\end{array}$ & $\begin{array}{l}\text { N, Hyp } \\
\text { d }\end{array}$ & ensi & HLP, $\mathrm{Hy}_{\mathrm{y}}$ & pide & $\mathrm{VD}$ & $\|-v \epsilon$ & dis & ;CE, & & \\
\hline
\end{tabular}

Moreover, the Spearman correlation also revealed that the hg38 level was significantly correlated with NIHSS scores $(r=$ 0.19; $95 \% \mathrm{Cl}$ : -0.041 to $0.409, p=0.04$ ) (Fig. 3. A). We did not find significant correlation between mmu and DLGAP4 expression level with NIHSS score. Spearman test showed a significant negative relationship between DLGAP4 level and $\mathrm{BMI}(\mathrm{r}=-0.21 ; 95 \% \mathrm{Cl}:-0.422$ to $0.0243, p=0.03)$ in IS patients (Fig. 3. B). Our results revealed the positive correlation between the level of DLGAP4 and HDL in IS patients ( $r=0.23$; $95 \% \mathrm{Cl}:-0.003$ to $0.440, p=0.02)$ (Fig. $3 \mathrm{C}$ ). 


\section{Diagnostic and prognostic value of circulating circRNAs (mmu- 015947, hg38-0008980, and DLGAP4) in IS}

ROC curve analysis showed the significant potential of mmu expression for diagnosing IS cases from the controls with an AUC of $0.775 \pm 0.039$ (95\% Cl: $0.697-0.853, p<0.0001)$ and the sensitivity and specificity were $80 \%$ and $72 \%$, respectively (Fig. 4A). The expression level of DLGAP4 and hg38 in 0-24 h after stroke also showed a nonsignificant diagnostic value for discriminating IS patients from the controls with AUC of 0.57 and 0.59 respectively (Fig. $4 \mathrm{~B}$ and C). The sensitivity and specificity were $78.67 \%$ and $38.67 \%$ for diagnostic value of DLGAP4 level and $56 \%$, and $60 \%$, for hg38 $(p>0.05)$.

In our study, we considered the mRS score of 3-6 in 6 months after stroke as an unfavorable functional outcome and mRS score of 0-2 as a favorable outcome. The mmu, DLGAP4, and hg38 levels showed no significant predictive prognosis for a 6-month unfavorable outcome relative to a favorable outcome with an AUC of $0.55,0.58$, and 0.55 respectively. These results demonstrated the expression levels of three circular RNA could not be identified as a functional outcome prediction marker for IS. The sensitivity and specificity for mmu, DLGAP4, and hg38 were as follows (86.54\% and $30.43 \%),(80.77 \%$ and $39.13 \%)$, and $(71.15 \%$ and $47.83 \%)$ respectively (Data not shown).

\section{Discussion}

There are still no clinical studies that identify circRNAs mmu-015947 and hg38-0008980 as biomarkers for diagnosis or progression of IS, therefore, our research was the first study that assessed circmmu and hg38 expression 0-24 hours after IS which showed that circ-mmu was up-regulated in IS patients and had a good value in predicting IS risk, circhg38 was decreased in patients compared with controls and logistic regression showed a significant association of its expression with IS risk. Moreover, we found a positive correlation between hg38 level and NIHSS. The expression level of hg38 was reduced significantly in peripheral blood of patients with SVD and the linear regression analysis showed a negative relationship between hg38 expression with SVD subtype.

CircDLGAP4 was decreased in IS patients compared with controls and its expression was negatively associated with IS risk. CircDLGAP4 had a negative correlation with BMI $(r=-0.2, p=0.03)$ and positive correlation with HDL $(r=0.23, p=$ 0.02).

Different pathways related to apoptosis, metabolism, and the immune system have been reported to be involved in the pathogenesis of ischemic-reperfusion injury $[26,27]$. The upregulation of circ-mmu-015947 in OGD/R HT22 cells and its association with all of these pathways was reported by Lin et al [7]. Circ-mmu-015947 may serve as a sponge" for 5 predicted target miRNAs

Such as mmu-miR-188-3p, mmu-miR-329-5p, mmu-miR-3057-3p, mmu-miR-5098, and mmu-miR-683. Thereby, mmu015947 inhibits the target miRNAs expression [7]. The expression of $\beta$-site amyloid precursor protein cleaving enzyme 1 (BACE1) is suppressed by up-regulated miR-188-3p expression thereby can attenuate neuroinflammation [28].

Dharap et al reported miR-329 could inhibit inflammation and neuronal death by suppressing the expression of peroxisome proliferator-activated receptor-gamma (PPARY) [29]. Therefore it seems that the elevated mmu-015947 expression may function as a neuroinflammation enhancer in IS pathophysiology. We found high expression of mmucircRNA-015947 in peripheral blood of 75 IS patients 0-24 $\mathrm{h}$ after stroke relative to controls. According to our results elevated mmu expression can increase the risk of IS (OR $=1.008,95 \% \mathrm{Cl}: 1.78-4.19, p<0.0001)$ and it also showed a significant diagnostic value with an AUC of 0.77 . In patients with hypertension, the mmu expression showed significant elevation compared with cases without hypertension while linear regression analysis did not show the significant relationships between mmu expression and hypertension. Hypertension is a risk factor for atherosclerotic diseases and IS [30]. Significant upregulation of mmu-015947 in IS patients with hypertension may increase the susceptibility to IS. In our 
study, there was no significant difference between the mean NIHSS score of patients with hypertension and patients without hypertension $(9.77 \pm 1.03$ vs $9.78 \pm 1.06)$ respectively. We did not find a significant Spearman correlation between mmu expression and NIHSS score in patients with hypertension and also in patients without hypertension (Data not shown). We found a non-significant positive correlation between mmu expression and stroke severity $(r=0.01, p=0.9)$. Probably, larger sample size may be needed to show the significant correlation between mmu level and NIHSS score.

In our study, a significant decrease in the expression level of circDLGAP4 was seen 0-24 $\mathrm{h}$ after IS. This result is consistent with previous studies that demonstrated the downregulation of circDLGAP4 and its protective effect in IS patients [8, 31]. We could not find the negative correlation between DLGAP4 expression and NIHSS score but logistic regression analysis showed the negative association between DLGAP4 level with risk of IS. This result was confirmed by the significant negative and positive correlation between BMI and HDL with DLGAP4 level respectively. Because the decrease in BMI and increase in HDL level decrease the risk of IS [32,33]. Our result showed patients with lower BMI and higher HDL relative to other patients had elevated DLGAP4 expression with a lower risk of IS.

In the zho study, the mean NIHSS score was $7.9 \pm 3.4$, while the mean NIHSS score in our patients was $9.8 \pm 0.74$.

Therefore, the value of circDLGAP4 might not be well correlated with NIHSS in patients with severe IS. 75 IS patients were enrolled in our study it seems that to reach a significant correlation between gene expression with stroke severity a larger sample is needed. The expression of circDLGAP4 showed a significant decrease relative to controls in our patients with SVD type. Cerebral small vessel disease has a significant relationship with type 2 diabetes mellitus [34, 35]. A previous study showed that circDLGAP4 promotes diabetic kidney disease injury by sponging miR-143 [36]. According to the abovementioned, there is a possibility of a correlation between DLGAP4-miR-143 and diabetes as well as SVD.

Circ-hg38-0008980 expression showed significant downregulation 0-24 h after stroke while logistic regression analysis represented a negative association with risk of IS. Subgroup analysis and Spearman test showed a significant positive correlation between Circ-hg38-0008980 level and NIHSS score moreover, linear regression has also shown the negative relationship between hg38 level and SVD stroke that was confirmed by the significant decrease in hg38 expression level relative to controls. We also found the lower mean NIHSS score in SVD patients relative to the others (7.96 \pm 1.09 vs $9.8 \pm$ $0.74)$ respectively. Therefore, it seems that hg38-0008980 level as a novel circRNA may have a positive correlation with stroke severity especially in patients with SVD.

\section{Conclusions}

This was the first study revealing the significant increase in the expression level of circ-mmu- 015947 and a significant decrease in hg38-0008980 expression in IS patients. Our results showed a significant positive correlation of hg38 with NIHSS and a significant diagnostic value of the circ-mmu-015947 expression after stroke. Larger sample size with more information needs to reach appropriate statistical power. A small number of IS patients with CE stroke had enrolled in our study; therefore, in this study, the value of circRNAs in patients with CE might not be well evaluated. To further clarify the role of circulating circular RNAs (mmu-015947, hg38-0008980, and DLGAP4) in IS, additional research with a larger sample size is needed to study the diagnostic and prognostic value as well as downstream effects of these circRNAs.

Further researches are necessary to finding the time-dependent changes in expression levels of these circRNAs after IS and their correlation with stroke severity. Taken together, circulating circ-mmu-015947 could serve as a novel biomarker for the diagnosis of IS, and the hg38-0008980 level showed a positive correlation with stroke severity. We found also the downregulation of circDLGAP4 after stroke while its expression showed a negative association with the risk of IS.

\section{Declarations}

\section{Acknowledgments}


The present study was derived from a thesis by Mastaneh Zeraatiannejad. This work was supported by Islamic Azad University, Zarghan Branch, Zarghan, Iran.

\section{Author contributions}

M.J. and A.B. designed this study. M.J. and M.Z. wrote the main manuscript text. M.B. edited the manuscript. A.B., and M.Z. collected all the clinical data. M.J., M.Z., and R.T. performed the statistical analysis. All authors have read and approved the final manuscript.

\section{Funding}

No financial assistance was received in support of the study.

\section{Data availability}

The datasets are available from the corresponding author upon reasonable request.

\section{Code availability}

Not applicable.

\section{Competing interests}

The authors declare that they have no competing interests.

\section{Ethics approval}

All methods were performed in accordance with the relevant guidelines and regulations. Ethics approval for this research was obtained by the local ethics committee of the Arsanjan Branch, Islamic Azad University, Iran (IR.IAU.A.REC.1399.029). The written informed consent form was obtained from all participants.

\section{Consent to participate}

Not applicable.

\section{Consent for publication}

Not applicable.

\section{References}

1. Tabrizi R, Lankarani KB, Kardeh B, Akbari H, Azarpazhooh MR, Borhani-Haghighi A (2021) A Comprehensive Systematic Review and Meta-analysis on the Risk Factors of Stroke in Iranian Population. Arch Iran Med 24(1):64-77 
2. Borhani-Haghighi A, Safari R, Heydari ST, Soleimani F, Sharifian M, Yektaparast Kashkuli S, Nayebi Khayatghuchani M, Azadi M, Shariat A, Safari A, Bagheri Lankarani K, Alshekhlee A, Cruz-Flores S (2013) Hospital mortality associated with stroke in southern iran. Iran J Med Sci 38(4):314-320

3. Anathhanam S, Hassan A (2017) Mimics and chameleons in stroke. Clin Med (Lond) 17(2):156-160. doi:10.7861/clinmedicine.17-2-156

4. Wang J, Zhao H, Fan Z, Li G, Ma Q, Tao Z, Wang R, Feng J, Luo Y (2017) Long noncoding RNA H19 promotes neuroinflammation in ischemic stroke by driving histone deacetylase 1-dependent M1 microglial polarization. Stroke 48(8):2211-2221

5. Chen L-L, Yang L (2015) Regulation of circRNA biogenesis. RNA Biol 12(4):381-388

6. Lu D, Xu A-D (2016) Mini review: circular RNAs as potential clinical biomarkers for disorders in the central nervous system. Front Genet 7:53

7. Lin S-P, Ye S, Long Y, Fan Y, Mao H-F, Chen M-T, Ma Q-J (2016) Circular RNA expression alterations are involved in OGD/R-induced neuron injury. Biochem Biophys Res Commun 471(1):52-56

8. Bai Y, Zhang Y, Han B, Yang L, Chen X, Huang R, Wu F, Chao J, Liu P, Hu G (2018) Circular RNA DLGAP4 ameliorates ischemic stroke outcomes by targeting miR-143 to regulate endothelial-mesenchymal transition associated with blood-brain barrier integrity. J Neurosci 38(1):32-50

9. Bao M-H, Szeto V, Yang BB, Zhu S-z, Sun H-S, Feng Z-P (2018) Long non-coding RNAs in ischemic stroke. Cell Death Dis $9(3): 1-12$

10. Lu D, Ho ES, Mai H, Zang J, Liu Y, Li Y, Yang B, Ding Y, Tsang CK, Xu A (2020) Identification of blood circular RNAs as potential biomarkers for acute ischemic stroke. Front NeuroSci 14:81

11. Ostolaza A, Blanco-Luquin I, Urdánoz-Casado A, Rubio I, Labarga A, Zandio B, Roldán M, Martínez-Cascales J, Mayor S, Herrera M (2020) Circular RNA expression profile in blood according to ischemic stroke etiology. Cell \& bioscience 10(1):1-12

12. Jarlstad Olesen MT, L SK (2021) Circular RNAs as microRNA sponges: evidence and controversies. Essays Biochem. doi:10.1042/EBC20200060

13. Liu C, Zhang C, Yang J, Geng X, Du H, Ji X, Zhao H (2017) Screening circular RNA expression patterns following focal cerebral ischemia in mice. Oncotarget 8(49):86535

14. Mehta SL, Pandi G, Vemuganti R (2017) Circular RNA expression profiles alter significantly in mouse brain after transient focal ischemia. Stroke 48(9):2541-2548

15. Bai Y, Zhang Y, Hua J, Yang X, Zhang X, Duan M, Zhu X, Huang W, Chao J, Zhou R (2016) Silencing microRNA-143 protects the integrity of the blood-brain barrier: implications for methamphetamine abuse. Sci Rep 6(1):1-15

16. Bai Y, Zhang Y, Han B, Yang L, Chen X, Huang R, Wu F, Chao J, Liu P, Hu G, Zhang JH, Yao H (2018) Circular RNA DLGAP4 Ameliorates Ischemic Stroke Outcomes by Targeting miR-143 to Regulate Endothelial-Mesenchymal Transition Associated with Blood-Brain Barrier Integrity. J Neurosci 38(1):32-50. doi:10.1523/JNEUROSCI.134817.2017

17. Erratum B et al "Circular RNA DLGAP4 Ameliorates Ischemic Stroke Outcomes by Targeting miR-143 to Regulate Endothelial-Mesenchymal Transition Associated with Blood-Brain Barrier Integrity" (2020).J Neurosci40 (44):8601. doi: 10.1523/JNEUROSCI.2320-20.2020

18. Yang R, Xu B, Yang B, Fu J, Liu L, Amjad N, Cai A, Tan C, Chen H, Wang X (2018) Circular RNA Transcriptomic Analysis of Primary Human Brain Microvascular Endothelial Cells Infected with Meningitic Escherichia coli. Mol Ther Nucleic Acids 13:651-664. doi:10.1016/j.omtn.2018.10.013

19. Powers WJ, Rabinstein AA, Ackerson T, Adeoye OM, Bambakidis NC, Becker K, Biller J, Brown M, Demaerschalk BM, Hoh B, Jauch EC, Kidwell CS, Leslie-Mazwi TM, Ovbiagele B, Scott PA, Sheth KN, Southerland AM, Summers DV, Tirschwell DL (2019) Guidelines for the Early Management of Patients With Acute Ischemic Stroke: 2019 Update to 
the 2018 Guidelines for the Early Management of Acute Ischemic Stroke: A Guideline for Healthcare Professionals From the American Heart Association/American Stroke Association. Stroke 50(12):e344-e418.

doi:10.1161/STR.0000000000000211

20. Unger T, Borghi C, Charchar F, Khan NA, Poulter NR, Prabhakaran D, Ramirez A, Schlaich M, Stergiou GS, Tomaszewski M, Wainford RD, Williams B, Schutte AE (2020) 2020 International Society of Hypertension Global Hypertension Practice Guidelines. Hypertension 75(6):1334-1357. doi:10.1161/HYPERTENSIONAHA.120.15026

21. American Diabetes A (2004) Diagnosis and classification of diabetes mellitus. Diabetes Care 27(Suppl 1):S5-S10. doi:10.2337/diacare.27.2007.s5

22. Williams LS, Yilmaz EY, Lopez-Yunez AM (2000) Retrospective assessment of initial stroke severity with the NIH Stroke Scale. Stroke 31(4):858-862. doi:10.1161/01.str.31.4.858

23. Nunn A, Bath PM, Gray LJ (2016) Analysis of the Modified Rankin Scale in Randomised Controlled Trials of Acute Ischaemic Stroke: A Systematic Review. Stroke Res Treat 2016:9482876. doi:10.1155/2016/9482876

24. Adams HP Jr, Bendixen BH, Kappelle LJ, Biller J, Love BB, Gordon DL, Marsh EE 3rd (1993) Classification of subtype of acute ischemic stroke. Definitions for use in a multicenter clinical trial. TOAST. Trial of Org 10172 in Acute Stroke Treatment. Stroke 24(1):35-41. doi:10.1161/01.str.24.1.35

25. Schmittgen TD, Livak KJ (2008) Analyzing real-time PCR data by the comparative C T method. Nat Protoc 3(6):1101

26. Zhang X, Chen L, Dang X, Liu J, Ito Y, Sun W (2014) Neuroprotective effects of total steroid saponins on cerebral ischemia injuries in an animal model of focal ischemia/reperfusion. Planta Med 80(8-9):637-644. doi:10.1055/s0034-1368584

27. Li P, Hu X, Gan Y, Gao Y, Liang W, Chen J (2011) Mechanistic insight into DNA damage and repair in ischemic stroke: exploiting the base excision repair pathway as a model of neuroprotection. Antioxid Redox Signal 14(10):1905-1918. doi:10.1089/ars.2010.3451

28. Zhang J, Hu M, Teng Z, Tang YP, Chen C (2014) Synaptic and cognitive improvements by inhibition of 2-AG metabolism are through upregulation of microRNA-188-3p in a mouse model of Alzheimer's disease. J Neurosci 34(45):14919-14933. doi:10.1523/JNEUROSCI.1165-14.2014

29. Dharap A, Pokrzywa C, Murali S, Kaimal B, Vemuganti R (2015) Mutual induction of transcription factor PPARgamma and microRNAs miR-145 and miR-329. J Neurochem 135(1):139-146. doi:10.1111/jnc.13220

30. Arboix A (2015) Cardiovascular risk factors for acute stroke: Risk profiles in the different subtypes of ischemic stroke. World J Clin Cases 3(5):418-429. doi:10.12998/wjcc.v3.i5.418

31. Zhu X, Ding J, Wang B, Wang J, Xu M (2019) Circular RNA DLGAP4 is down-regulated and negatively correlates with severity, inflammatory cytokine expression and pro-inflammatory gene miR-143 expression in acute ischemic stroke patients. Int J Clin Exp Pathol 12(3):941-948

32. Reina SA, Llabre MM, Allison MA, Wilkins JT, Mendez AJ, Arnan MK, Schneiderman N, Sacco RL, Carnethon M, Delaney JA (2015) HDL cholesterol and stroke risk: The Multi-Ethnic Study of Atherosclerosis. Atherosclerosis 243(1):314-319. doi:10.1016/j.atherosclerosis.2015.09.031

33. Mitchell AB, Cole JW, McArdle PF, Cheng YC, Ryan KA, Sparks MJ, Mitchell BD, Kittner SJ (2015) Obesity increases risk of ischemic stroke in young adults. Stroke 46(6):1690-1692. doi:10.1161/STROKEAHA.115.008940

34. Liu J, Rutten-Jacobs L, Liu M, Markus HS, Traylor M (2018) Causal Impact of Type 2 Diabetes Mellitus on Cerebral Small Vessel Disease: A Mendelian Randomization Analysis. Stroke 49(6):1325-1331. doi:10.1161/STROKEAHA.117.020536

35. Sanahuja J, Alonso N, Diez J, Ortega E, Rubinat E, Traveset A, Alcubierre N, Betriu A, Castelblanco E, Hernandez M, Purroy F, Arcidiacono MV, Jurjo C, Fernandez E, Puig-Domingo M, Groop PH, Mauricio D (2016) Increased Burden of Cerebral Small Vessel Disease in Patients With Type 2 Diabetes and Retinopathy. Diabetes Care 39(9):1614-1620. doi:10.2337/dc15-2671

Page $13 / 17$ 
36. Bai S, Xiong X, Tang B, Ji T, Li X, Qu X, Li W (2020) Exosomal circ_DLGAP4 promotes diabetic kidney disease progression by sponging miR-143 and targeting ERBB3/NF-kappaB/MMP-2 axis. Cell Death Dis 11(11):1008. doi:10.1038/s41419-020-03169-3

\section{Figures}

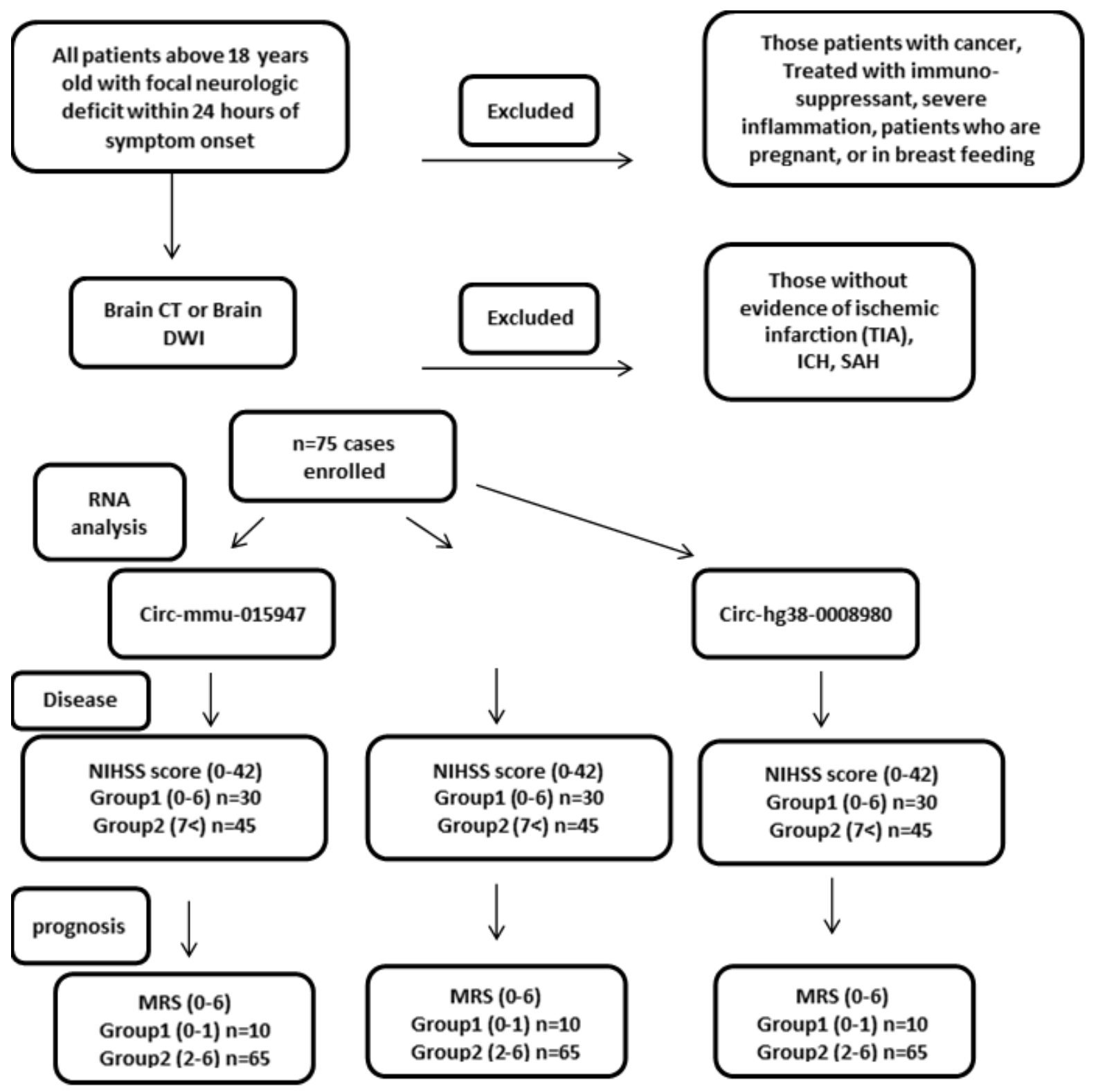

Figure 1

Flowchart for screening, exclusion and enrolment of the study participants. 

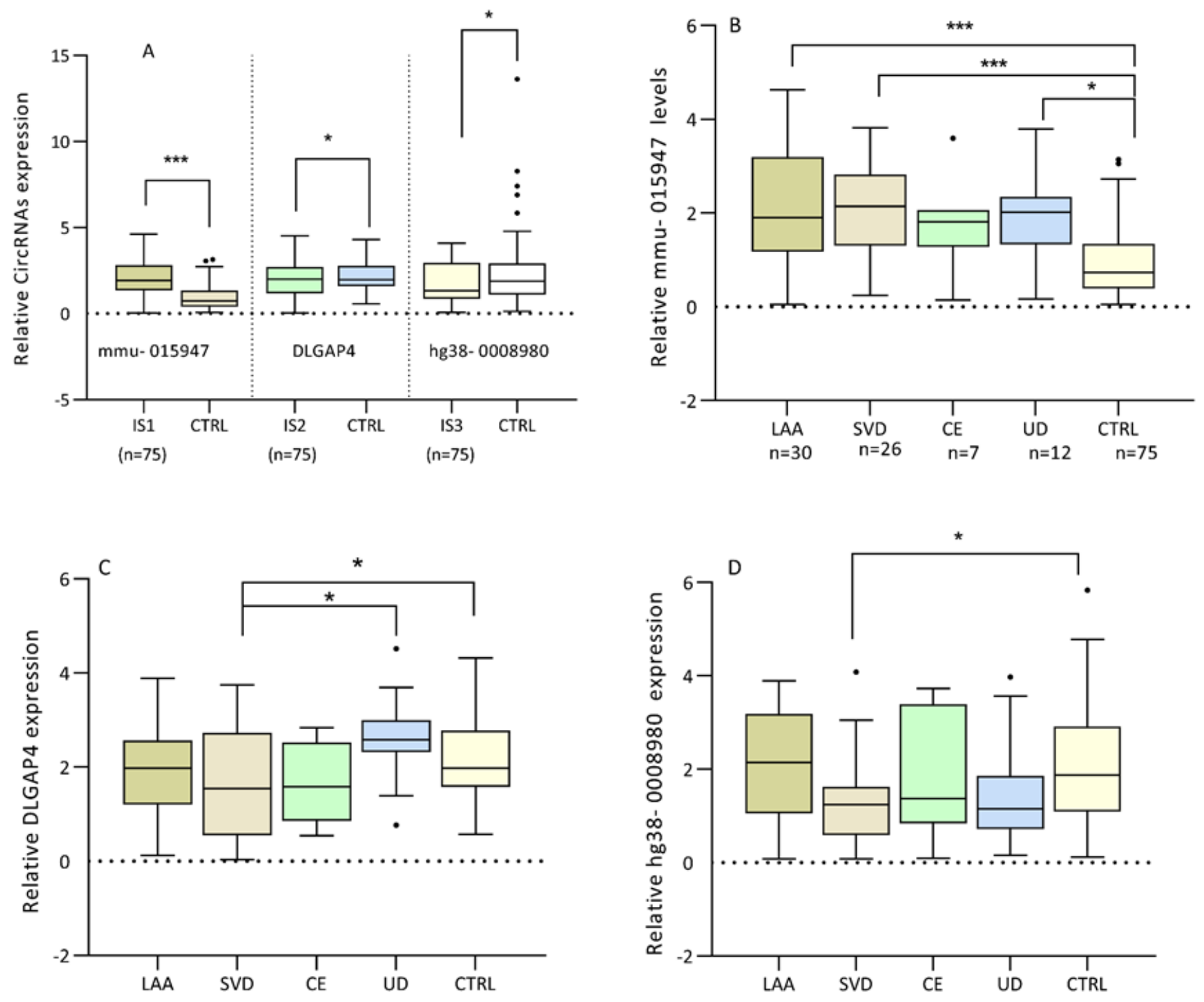

Figure 2

The expression levels of mmu-015947, hg38-0008980, and DLGAP4 in patients, controls, and different subtypes of ischemic stroke. (A) Independent Student's t-test revealed that mmu-015947 expression in IS patients were significantly higher than those in controls while in IS cases hg38-0008980 and DLGAP4 levels were lower than controls. (B,C,and D) The comparison of mmu-015947, hg38-0008980, and DLGAP4 levels in different subtypes of IS by One-Way ANOVA analysis. Results were expressed as mean \pm SEM. . ${ }^{*} p<0.05$. ${ }^{\star \star *} p<0.001$. Abbreviations: IS, ischemic stroke; CTRL,control; LAA, large-artery atherosclerosis; SVD, small-vessel disease; CE, cardioembolism; UD, undetermined 

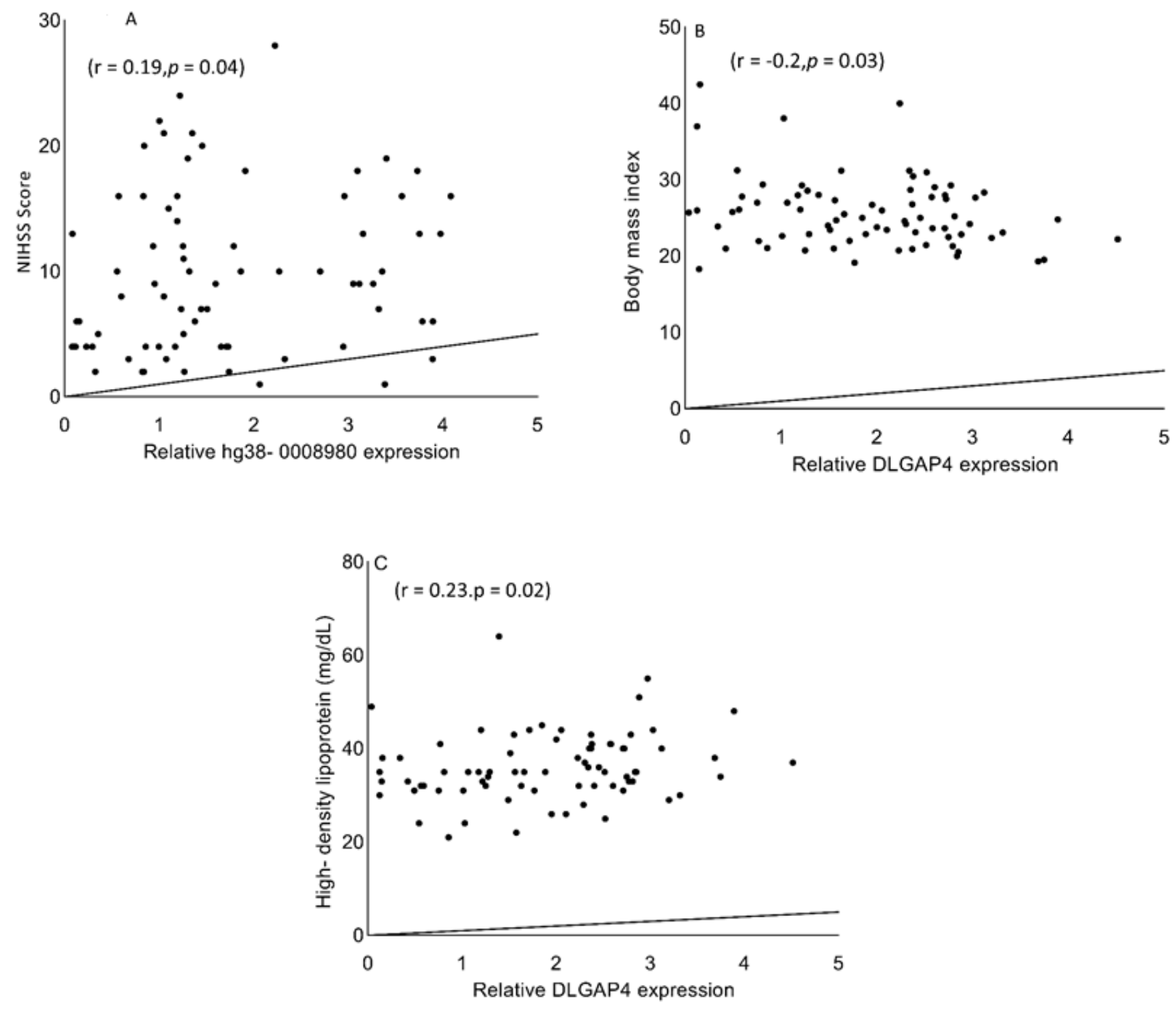

Figure 3

The Spearman Correlation between expression levels of circRNAs with NIHSS and BMI in patients.

(A) The Spearman Correlation between expression of hg38-0008980 and NIHSS in patients (B) Correlation between expression of DLGAP4 and BMI (C) Correlation between expression of DLGAP4 and HDL. Abbreviations: NIHSS, National Institutes of Health Stroke Scale; BMI, body mass index; HDL, high-density lipoprotein 

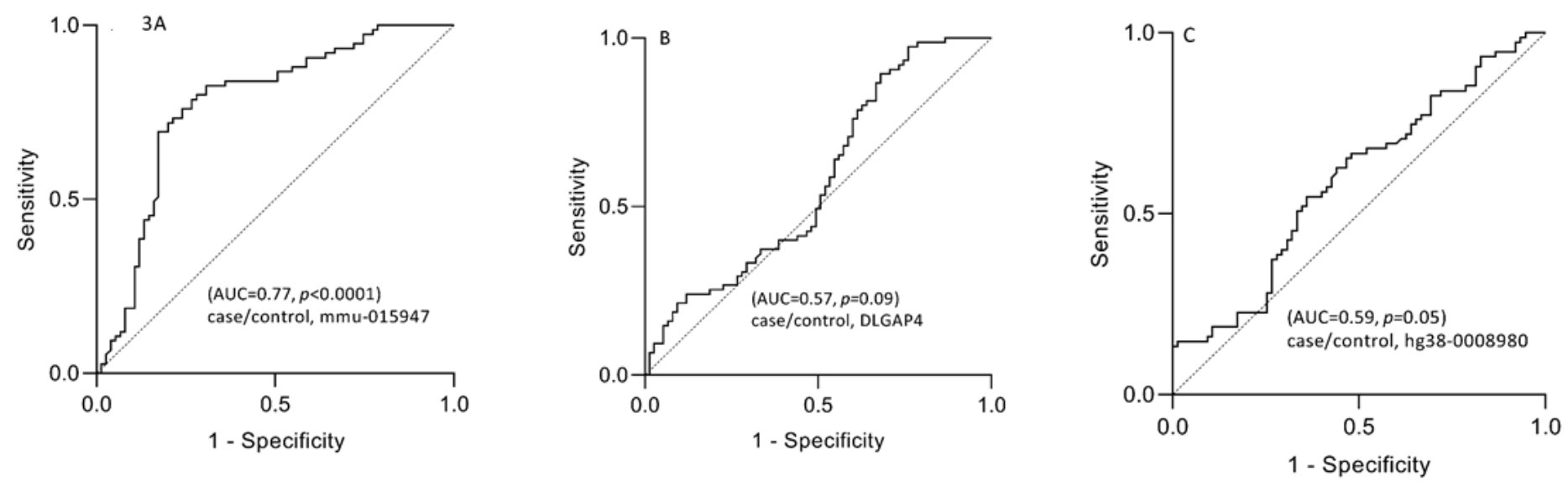

\section{Figure 4}

ROC curves. (A, B, and C) ROC curve analyses of mmu-015947, hg38-0008980, and DLGAP4 for discriminating IS patients from the controls. Abbreviations: ROC, receiver operating characteristic; AUC, area under the curve 